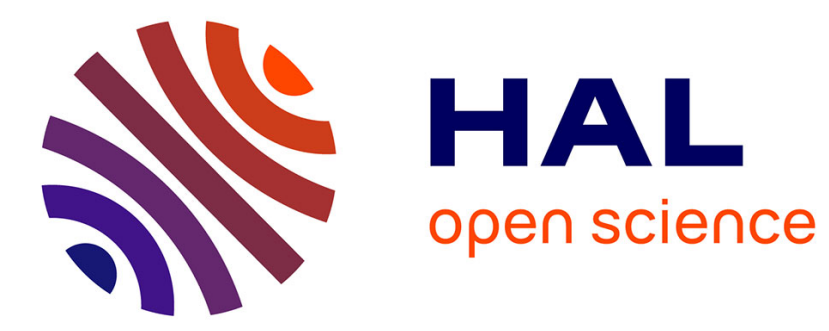

\title{
Korn-Poincaré inequalities for functions with a small jump set
}

Antonin Chambolle, Sergio Conti, Gilles Francfort

\section{To cite this version:}

Antonin Chambolle, Sergio Conti, Gilles Francfort. Korn-Poincaré inequalities for functions with a small jump set. 2014. hal-01091710

\section{HAL Id: hal-01091710 https://hal.science/hal-01091710}

Preprint submitted on 7 Dec 2014

HAL is a multi-disciplinary open access archive for the deposit and dissemination of scientific research documents, whether they are published or not. The documents may come from teaching and research institutions in France or abroad, or from public or private research centers.
L'archive ouverte pluridisciplinaire HAL, est destinée au dépôt et à la diffusion de documents scientifiques de niveau recherche, publiés ou non, émanant des établissements d'enseignement et de recherche français ou étrangers, des laboratoires publics ou privés. 


\section{Korn-Poincaré inequalities for functions with a small jump set}

December 5, 2014

Antonin Chambolle, ${ }^{1}$ Sergio Conti ${ }^{2}$ and Gilles Francfort ${ }^{3}$

${ }^{1}$ CMAP, Ecole Polytechnique, CNRS

91128 Palaiseau Cedex, France

2 Institut für Angewandte Mathematik, Universität Bonn

53115 Bonn, Germany

3 Laboratoire Analyse, Géométrie et Applications, Université Paris-Nord, CNRS

93430 Villetaneuse, France

Functions in $S B D^{p}$ arise naturally in the study of geometrically linear fracture models. They have a jump set of finite $(n-1)$-dimensional measure and, away from the jump set, a symmetrized gradient $e(u)=\left(\nabla u+\nabla u^{T}\right) / 2$ in $L^{p}, p \geq 1$. We show that if the measure of the jump set is sufficiently small with respect to the size of the domain, then the function $u$ can be approximated by an affine function away from a small exceptional set, with an error which depends solely on $e(u)$. We also derive a corresponding trace statement.

\section{Introduction}

Functions of bounded deformation have been introduced to study plasticity, damage and fracture models in a geometrically linear setting [Suq78, Tem83, TS81, AG80, KT83]. The space $B D(\Omega)$ is the set of functions $u \in L^{1}\left(\Omega ; \mathbb{R}^{n}\right)$ such that the symmetric part of the distributional gradient $E u=\left(D u+D u^{T}\right) / 2$ is a bounded measure. They share many properties with the functions of bounded variation, for example the strain can be decomposed in a part absolutely continuous with respect to the Lebesgue measure $\mathcal{L}^{n}$, a jump part and an intermediate, so-called Cantor part,

$$
E u=e(u) \mathcal{L}^{n}+\frac{[u] \otimes n+n \otimes[u]}{2} \mathcal{H}^{n-1}\left\llcorner J_{u}+E^{c} u\right.
$$

where the jump set $J_{u}$ is a $(n-1)$-rectifiable subset of $\Omega,[u]: J_{u} \rightarrow \mathbb{R}^{n}$ denotes the jump of $u$, and $n$ the normal to $J_{u}$, see [ACD97]. The three terms in (1.1) are mutually orthogonal and have a clearly distinct physical 
interpretation: $e(u)$ represents the regular strain, $[u]$ the crack opening or the plastic slips, $E^{c} u$ some notion of diffuse damage.

The modeling of fracture in linear elasticity[FM98, BFM08] focuses on the interplay between the regular and the jump part, and is normally restricted to the special functions of bounded deformation $S B D$, defined as those $u \in B D$ for which $E^{c} u=0$, see also [Cha03, SFO08, FI14, Iur14]. In fracture models it is natural to relate $\|e(u)\|_{L^{2}}$ to an elastic energy and the total area of the crack $\mathcal{H}^{n-1}\left(J_{u}\right)$ to the fracture energy, considering functionals of the type $\|e(u)\|_{L^{2}}^{2}+\mathcal{H}^{n-1}\left(J_{u}\right)$ which constitute the vectorial counterpart to the Mumford-Shah functional from image segmentation [AFP00]. This leads to the study of the space $S B D^{p}(\Omega)$, which is defined as the set of $u \in B D(\Omega)$ such that $E^{c} u=0, e(u) \in L^{p}$ and $\mathcal{H}^{n-1}\left(J_{u}\right)<\infty$, see [BCDM98, Cha04, Cha05]. The key additional difficulty with respect to scalar models based on functions of bounded variation is the lack of control on the skew-symmetric part of the distributional gradient, $D u-D u^{T}$.

Korn's inequality is one key ingredient in the study of linear elasticity and of functions of bounded deformation. In its standard version it states that, if $u \in W^{1, p}\left(\Omega ; \mathbb{R}^{n}\right)$ with $p \in(1, \infty)$ and $\Omega \subset \mathbb{R}^{n}$ a bounded, connected Lipschitz set, then there is $A \in \mathbb{R}^{n \times n}$ such that

$$
\|D u-A\|_{L^{p}(\Omega)} \leq c(p, \Omega)\left\|D u+D u^{T}\right\|_{L^{p}(\Omega)},
$$

see for example [Reš70, Tin72] or [Nit81] for a proof. This has been generalized to many different settings, including the geometrically nonlinear case [FJM02], mixed growth [CDM14] and incompatible strain fields which contain dislocations [NPW12, MSZ14].

Korn's inequality (1.2) does not hold for $p=1$, and indeed one can construct functions such that $D u+D u^{T} \in L^{1}$ but $D u \notin L^{1}$, see [Orn62, CFM05, KK11]. Correspondingly, $B D$ is not a subset of $B V$, in the sense that for any open set $\Omega \subset \mathbb{R}^{n}$ there are functions $u \in L^{1}\left(\Omega ; \mathbb{R}^{n}\right)$ such that $D u+D u^{T}$ is a bounded measure, but $D u$ is not.

A combination of Poincaré's and Sobolev inequalities with Korn's inequality (1.2) leads to estimates on $u(x)-A x-b$ in $L^{q}, q \geq 1$. Although Korn's inequality fails for $p=1$, the combination with the inequalities in which the derivative is lost is still true. Indeed, for $u \in B D(\Omega)$ there are $A \in \mathbb{R}^{n \times n}$ and $b \in \mathbb{R}^{n}$ such that

$$
\|u(x)-(A x+b)\|_{L^{1 *}(\Omega)} \leq c(\Omega)|E u|(\Omega),
$$

see [Koh82, Tem83]. Here $1^{*}=n /(n-1)$ is the Sobolev conjugate exponent to 1 , and $\Omega \subset \mathbb{R}^{n}$ is again a bounded, connected Lipschitz set. 


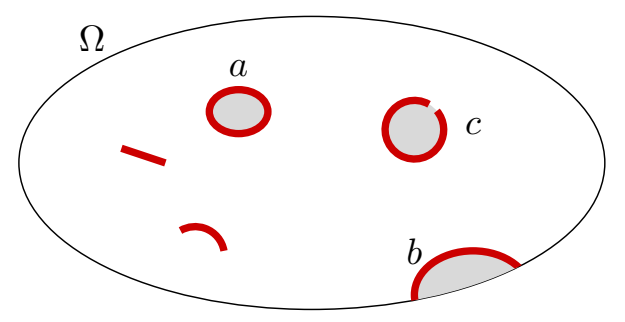

Figure 1: Sketch of the geometry. The jump set is short with respect to the diameter of the domain $\Omega$ and cannot cut it into two "large" blocks. However, small regions can be disconnected from the rest, either inside $(a)$ or close to the boundary $(b)$; also in regions which are "almost" disconnected from the majority region $(c)$ one cannot obtain a uniform estimate.

For functions in $S B D^{p}$ one may expect a stronger result to hold, since a stronger control of the gradient is present. From a physical viewpoint, we consider a sample which contains small fractures, as illustrated in Figure 1. If the total $(n-1)$-dimensional area of the fracture is small, most of the material still belongs to one large piece, whose deformation is controlled only by the regular strain $e(u)$. Small subsets can however be completely detached, therefore we cannot expect an estimate in the entire set $\Omega$, but only in a subset $\Omega \backslash \omega$. The "holes" $\omega$ are the parts of $\Omega$ which are effectively separated from the rest by $J_{u}$. For their volume we obtain first the simpler estimate $\mathcal{L}^{n}(\omega) \leq c \mathcal{H}^{n-1}\left(J_{u}\right) \operatorname{diam}(\Omega)$ in Section 2 and then the optimal isoperimetric estimate $\mathcal{L}^{n}(\omega) \leq c\left(\mathcal{H}^{n-1}\left(J_{u}\right)\right)^{n /(n-1)}$ in Section 4. Further, a corresponding trace estimate can be obtained, see Section 4 . Our bound on $u$ in $L^{q}$ with $q=p n /(n-1)$ only reaches the optimal exponent of the full Korn-Sobolev inequality (1.3) if $p=1$.

The key idea of the proof is to use the fundamental theorem of calculus along lines which do not intersect the jump set to estimate the variation of $u$. This would be immediate in the $B V$ setting, in which one fully controls the gradient. In a $B D$ setting one only obtains control of the longitudinal component of $u$. Therefore one needs to consider lines with many different orientations, making sure to choose them so that they do not intersect the jump set, a strategy that was used for proving density in [Cha04, Cha05] and for proving rigidity in one and multiwell settings for example in [Koh82, DM95, CS06]. More details are explained in the introduction to Section 2.

Our main result is the following.

Theorem 1. Let $\Omega \subset \mathbb{R}^{n}$ be a connected bounded Lipschitz set, $p \in[1, \infty)$. There is a constant $c$ which depends only on $p$ and $\Omega$ such that for any 
$u \in S B D(\Omega)$, one can find Borel sets $\omega \subset \Omega$ and $\omega_{\Gamma} \subset \partial \Omega$ with

$$
\mathcal{L}^{n}(\omega) \leq c\left(\mathcal{H}^{n-1}\left(J_{u}\right)\right)^{n /(n-1)} \text { and } \mathcal{H}^{n-1}\left(\omega_{\Gamma}\right) \leq c \mathcal{H}^{n-1}\left(J_{u}\right)
$$

and an affine function $a: \mathbb{R}^{n} \rightarrow \mathbb{R}^{n}$ with $D a+D a^{T}=0$ such that, with $q=p n /(n-1)$

$$
\int_{\Omega \backslash \omega}|u-a|^{q} d x \leq c\left(\int_{\Omega}|e(u)|^{p} d x\right)^{n /(n-1)}
$$

and

$$
\int_{\partial \Omega \backslash \omega_{\Gamma}}|T u-a|^{p} d \mathcal{H}^{n-1} \leq c \int_{\Omega}|e(u)|^{p} d x .
$$

Here $e(u)$ denotes the part of the strain $E u=\left(D u+D u^{T}\right) / 2$ which is absolutely continuous with respect to $\mathcal{L}^{n}$ and Tu denotes the trace of $u$ on $\partial \Omega$.

From the statement it is clear that the assertion is only relevant if $u \in$ $S B D^{p}$ for some $p \geq 1$; the case $p=1$ is included.

The original motivation for this work was in the study of a fracture model with a non-interpenetration constraint, which will be discussed elsewhere $[\mathrm{CCF}]$. A different application, where the trace estimate is specifically used, arises in the study of pattern formation in delaminated thin films, see [BCM].

A natural question is whether a similar estimate for $\nabla u$ can be derived. We do not know if this is true and, in particular, we do now know if $S B D^{p}$, $p>1$, is a subset of $B V$.

The rest of this paper contains the proof of Theorem 1. In Section 2 we construct the affine map $a$ with suboptimal estimates on both $u$ and $|\omega|$. In Section 3 we build upon this result to prove higher integrability of $u$ in the interior. In Section 4 we refine the argument of Section 3 to prove higher integrability up to the boundary and the trace estimate; the proof of Theorem 1 then follows easily by covering.

Notation. As stated above, for $\Omega \subset \mathbb{R}^{n}$ open $B D(\Omega)$ is the set of functions $u \in L^{1}$ such that $E u=\left(D u+D u^{T}\right) / 2$ is a bounded measure. Then $E u$ obeys (1.1), and $S B D$ is the subset of $B D$ such that $E^{c} u=0$. We denote by $e(u)$ the density of $E u$ with respect to $\mathcal{L}^{n}$, as in (1.1).

We shall use the results on slicing of $B D$ functions from [ACD97]. In particular, let $u \in S B D, \xi \in S^{n-1}$. We set $u_{y}^{\xi}(t)=\xi \cdot u(y+t \xi)$. By the structure theorem [ACD97, Th. 4.5], for almost every $y$ one has: $u_{y}^{\xi} \in S B V$, $d u_{y}^{\xi} / d t=\xi e(u) \xi \mathcal{L}^{1}$-a.e., and the section of the jump set is the jump set of $u_{y}^{\xi}$. In particular we have that, for a.e. $x$ such that $x+[0,1] \xi \subset \Omega \backslash J_{u}$,

$$
\xi \cdot u(x)-\xi \cdot u(x+\xi)=\int_{0}^{1} \xi \cdot e(u)(x+t \xi) \xi d t .
$$


Functions in $B D$ have an $L^{1}$ trace on the boundary, see [Suq79, TS81, Bab13], which we denote by $T u$.

Throughout the paper $c$ denotes a generic constant, which may change from line to line, and which, unless otherwise stated, depends only on the spatial dimension $n$ and on the integrability exponent $p$.

Acknowledgements The authors gratefully acknowledge the hospitality of the Mathematisches Forschungsinstitüt Oberwolfach (MFO) where this research was initiated. A. Chambolle is partially funded by the Agence Nationale de la Recherche, project ANR-12-BS01-0014-01 "GEOMETRYA".

\section{The local estimate}

We start proving the Korn-Poincaré estimate in a square, with an exceptional set whose volume is proportional to $\mathcal{H}^{n-1}\left(J_{u}\right)$. This is the part of the proof where we construct the affine map $a$. Before stating our result we give a sketch of the main ideas.

The key strategy is to use the fundamental theorem of calculus along segments which do not intersect the jump set, in order to relate the values of $u$ at different points. Since we only control the strain and not the full gradient we need to focus on the longitudinal component, and with enough regularity we compute

$$
\frac{d}{d t} \xi \cdot u(x+t \xi)=\xi \cdot D u(x+t \xi) \xi=\xi \cdot \frac{D u+D u^{T}}{2}(x+t \xi) \xi .
$$

We shall use this to relate the values of $u$ in $n+1$ points, called $\left\{z_{0}, \ldots, z_{n}\right\}$, which form the vertices of an $n$-dimensional simplex, see Figure 2. Precisely, integrating (2.1) gives a control on the longitudinal variations,

$$
\mid\left(z_{i}-z_{j}\right) \cdot\left(u\left(z_{i}\right)-u\left(z_{j}\right)|\leq| z_{i}-z_{j}\left|\int_{\left[z_{i}, z_{j}\right]}\right| e(u) \mid d \mathcal{H}^{1}\right.
$$

whenever the restriction of $u$ to the line through $z_{i}$ and $z_{j}$ belongs to $W^{1,1}$. We shall use the $n+1$ values $u\left(z_{i}\right)$ to define a linear map $\tilde{a}: \mathbb{R}^{n} \rightarrow \mathbb{R}^{n}$ with $\tilde{a}\left(z_{i}\right)=u\left(z_{i}\right)$. For this map as well, the longitudinal variations are related to the components of the symmetrized gradient. The longitudinal variations are those of $u$, hence they remain small. Therefore, since $\tilde{a}$ is affine, the symmetric part of the gradient of $\tilde{a}$ is also bounded by $\|e(u)\|_{L^{1}}$. We can then replace $\tilde{a}$ by its projection on affine functions with skew-symmetric gradients. 


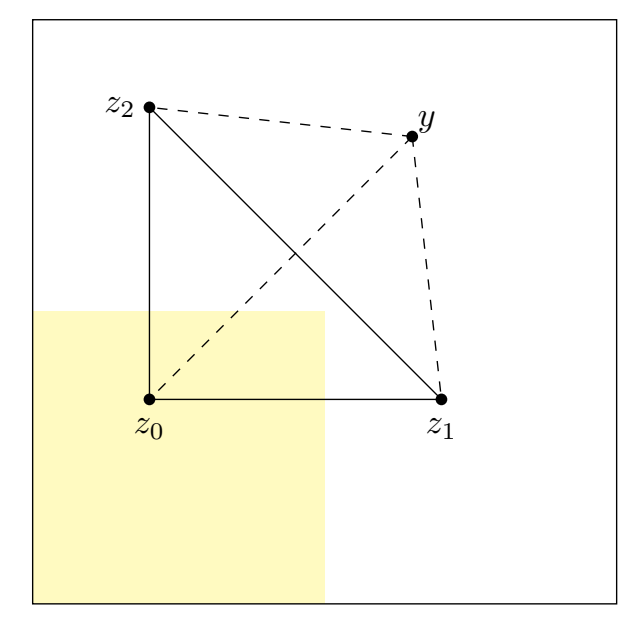

Figure 2: Sketch of the construction in Proposition 2. We first obtain rigidity on the vertices $z_{i}=z_{0}+t_{*} e_{i}$ of a simplex, and then show that the same rigidity is inherited by the other points $y \in Q$.

This strategy works under three assumptions: that the segments $\left[z_{i}, z_{j}\right]$ do not intersect the jump set, that we can use the fundamental theorem of calculus along these segments, and that the $L^{1}$ norm of $e(u)$ on the segments can be estimated by the $L^{1}$ norm on the entire set. The first and the third are true "on average", if we average over sets of parallel segments first, and therefore for many possible choices of the $z_{i}$. The second one is true by the structure theorem for almost every choice.

It remains to show that $u$ is close to $a$ on a large part of the square. To do this we consider a generic point $y$ and repeat the above argument on the segments $\left[y, z_{i}\right]$. This leads to an estimate on the $n+1$ longitudinal components of $(u-a)(y)$ in terms of the $L^{1}$ norm of $e(u)$ along those segments. A simple argument from convex analysis shows that these components are linearly independent in a uniform way, permitting to estimate $|u-a|(y)$ from them. Finally we need to make sure that we can use the fundamental theorem of calculus for many points $y$ and that the integral of $e(u)$ along the segments $\left[y, z_{i}\right]$ can be estimated by the $L^{1}$ norm of $e(u)$. Both require additional conditions on the choices of the point $z_{0}$, see Figure 3 . The final choice will have to fulfill the four conditions given in (2.8), (2.9), (2.12), and (2.17).

Proposition 2. Let $u \in S B D\left(Q_{r}\right), Q_{r}=(-r, r)^{n}, p \in[1, \infty)$. Then there are $\omega \subset Q_{r}$ with

$$
\mathcal{L}^{n}(\omega) \leq c r \mathcal{H}^{n-1}\left(J_{u}\right)
$$




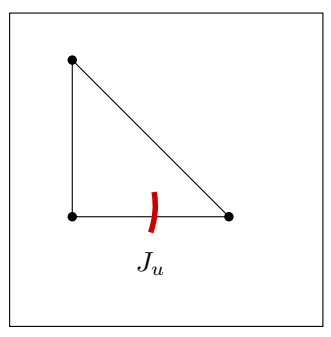

(a)

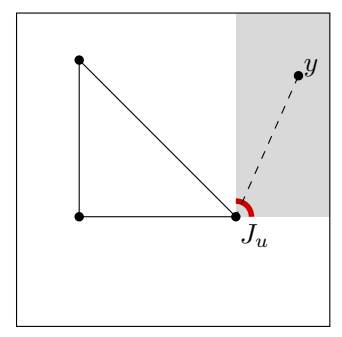

(b)

Figure 3: Two possible difficulties in Proposition 2. (a): In choosing the simplex we need to make sure that the jump set does not intersect its edges. (b): Since the jump set will "shadow" points $y$ with $\left[y, z_{i}\right] \cap J_{u} \neq \emptyset$, we need to make sure that we choose the simplex so that no larger part of $J_{u}$ is close to its vertices. Analogously $e(u)$ should not concentrate on the edges of the simplex and around the vertices.

and an affine function $a: \mathbb{R}^{n} \rightarrow \mathbb{R}^{n}$ with $e(a)=0$ such that

$$
\int_{Q_{r} \backslash \omega}|u-a|^{p} d x \leq c r^{p} \int_{Q_{r}}|e(u)|^{p} d x
$$

The constant depends only on $n$ and $p$.

Proof. By rescaling we can assume $r=1$; we write for brevity $Q=Q_{1}$. Instead of finding $a$ with $e(a)=0$ it suffices to construct an affine function $a$ with $|e(a)| \leq c\|e(u)\|_{L^{1}}$, which will be proven in (2.11), and then to replace $a$ by its projection on infinitesimal rotations. Further, we can assume

$$
\mathcal{H}^{n-1}\left(J_{u}\right) \leq \frac{1}{32 n^{3}} .
$$

Indeed, if not, one can simply take $\omega=Q$ and $a=0$ with $c=32 \cdot n^{3}$. We subdivide the proof in several steps.

Step 1: Fundamental theorem of calculus along segments.

We consider for $(x, \xi, t) \in Q \times S^{n-1} \times \mathbb{R}$ the relation

$$
\xi \cdot(u(x+t \xi)-u(x))=t \int_{0}^{1} \xi \cdot e(u)(x+s t \xi) \xi d s .
$$

If $u \in C^{1}\left(Q ; \mathbb{R}^{n}\right)$ this would hold for all $(x, \xi, t)$ with $x+t \xi \in Q$. Here we need to pay attention to both to the jump set and to the possible lack of pointwise 
values and differentiability on the appropriate null sets. To do this we define the characteristic function of the exceptional set $T: \mathbb{R}^{n} \times S^{n-1} \times \mathbb{R} \rightarrow \mathbb{R}$ by

$$
T(x, \xi, t)= \begin{cases}1 & \text { if } x \in Q, x+t \xi \in Q \text { and }(2.5) \text { does not hold } \\ 0 & \text { otherwise. }\end{cases}
$$

Both sides in (2.5) are measurable, hence $T$ is measurable. We inserted the condition $x, x+t \xi \in Q$ since we are only interested in points inside $Q$. The condition "(2.5) does not hold" includes the case in which the integral is not defined.

By the structure theorem [ACD97, Th. 4.5], for any fixed $\xi$ the following holds: for almost every $x \in Q$ the section $t \mapsto \xi \cdot u(x+t \xi)$ is in $S B V$, its jump set coincides almost everywhere with the section of the jump set of $u$, and its derivative is $\xi \cdot e(u) \xi$. This means that for $\mathcal{L}^{n}$-almost every $x \in Q$ one of these two options holds: either $x+\mathbb{R} \xi$ intersects $J_{u}$ or $(2.5)$ holds for $\mathcal{L}^{1}$-almost every $t$ in the relevant interval. Since, for any $\xi \in S^{n-1}$, the set

$$
\omega_{\xi}=\left\{x \in Q: x+\mathbb{R} \xi \cap J_{u} \neq \emptyset\right\}
$$

has measure bounded by $\left|\omega_{\xi}\right| \leq \mathcal{H}^{n-1}\left(J_{u}\right) \operatorname{diam}(Q)=2 \sqrt{n} \mathcal{H}^{n-1}\left(J_{u}\right)$, we obtain the estimate

$$
\int_{\mathbb{R}^{n}} \int_{\mathbb{R}} T(x, \xi, t) d t d x \leq\left|\omega_{\xi}\right| \operatorname{diam}(Q) \leq 4 n \mathcal{H}^{n-1}\left(J_{u}\right) \quad \text { for any } \xi \in S^{n-1} .
$$

We need now to choose the point $z_{0}$ as well as $n$ other reference points, obtained from the point $z_{0}$ by shifts along the canonical basis, and we need to control also their interactions, see Figure 2. Therefore we define, for $z_{0} \in q=(-1,0)^{n} \subset Q$ and $t \in(1 / 2,1)$,

$$
G\left(z_{0}, t\right)=\sum_{i=1}^{n} T\left(z_{0}, e_{i}, t\right)+\sum_{i=1}^{n} \sum_{j=i+1}^{n} T\left(z_{0}+t e_{i}, \frac{e_{j}-e_{i}}{\sqrt{2}}, t \sqrt{2}\right) .
$$

Integrating and using (2.7) on each term leads to

$$
\begin{aligned}
\int_{1 / 2}^{1} \int_{q} G\left(z_{0}, t\right) d z_{0} d t & \leq \int_{\mathbb{R}} \int_{\mathbb{R}^{n}} G d z_{0} d t \\
& \leq 4 n^{2} \mathcal{H}^{n-1}\left(J_{u}\right)+\frac{n(n-1)}{2} \frac{4 n}{\sqrt{2}} \mathcal{H}^{n-1}\left(J_{u}\right) \leq 4 n^{3} \mathcal{H}^{n-1}\left(J_{u}\right) .
\end{aligned}
$$

We choose $t_{*} \in(1 / 2,1)$ at a set value for the rest of the proof, such that $\int_{q} G\left(z_{0}, t_{*}\right) d z_{0} \leq 8 n^{3} \mathcal{H}^{n-1}\left(J_{u}\right)$. Therefore, (2.4) implies that we can have 
$G\left(z_{0}, t_{*}\right) \neq 0$ only for at most one-quarter of the values of $z_{0} \in q$, and therefore the statement

$$
\begin{array}{r}
(2.5) \text { holds on all edges }\left[z_{0}, z_{0}+t_{*} e_{i}\right], i=1, \ldots, n \\
\text { and }\left[z_{0}+t_{*} e_{i}, z_{0}+t_{*} e_{j}\right], 1 \leq i<j \leq n
\end{array}
$$

is true for three-quarters of the values of $z_{0} \in q$. The choice of $z_{0}$ among these possibilities will occur later.

Step 2: Estimate for e(u) along the edges of the simplex, construction of a.

For $t_{*}$ as above and any $z_{0} \in q$ we set $z_{i}=z_{0}+t_{*} e_{i} \in Q, i=1, \ldots, n$, and define

$$
F\left(z_{0}\right)=\sum_{i, j=0}^{n} \int_{\left[z_{i}, z_{j}\right]}|e(u)| d \mathcal{H}^{1} .
$$

We integrate in $z_{0}$, change variables and the order of integration to obtain (setting $e_{0}=0$ )

$$
\begin{aligned}
\int_{q} F\left(z_{0}\right) d z_{0} & =\sum_{i, j=0}^{n} \int_{q} \int_{0}^{t_{*}}|e(u)|\left(z_{0}+t_{*} e_{i}+s\left(e_{j}-e_{i}\right)\right)\left|e_{i}-e_{j}\right| d s d z_{0} \\
& \leq \sqrt{2} \sum_{i, j=0}^{n} \int_{0}^{t_{*}} \int_{Q}|e(u)|(x) d x d s \\
& \leq \sqrt{2}(n+1)^{2}\|e(u)\|_{L^{1}(Q)} .
\end{aligned}
$$

Therefore for three-quarters of the choices of $z_{0} \in q$ one has

$$
F\left(z_{0}\right) \leq 4 \sqrt{2}(n+1)^{2}\|e(u)\|_{L^{1}(Q)} .
$$

This is the second requirement on $z_{0}$, and we still have at least half the volume of $q$ at our disposal to enforce additional conditions later.

We consider any choice of $z_{0}$ such that both properties (2.8) and (2.9) hold. Then (2.5) gives

$$
\left|\left(e_{i}-e_{j}\right) \cdot\left(u\left(z_{i}\right)-u\left(z_{j}\right)\right)\right| \leq c\|e(u)\|_{L^{1}(Q)}, \quad i, j=0, \ldots, n .
$$

We define the affine map $a: \mathbb{R}^{n} \rightarrow \mathbb{R}^{n}$ by setting $a\left(z_{i}\right)=u\left(z_{i}\right), i=0, \ldots, n$. From (2.10) we obtain

$$
t_{*}\left|(D a)_{i i}\right|=\left|e_{i} \cdot\left(a\left(z_{0}+t_{*} e_{i}\right)-a\left(z_{0}\right)\right)\right| \leq c\|e(u)\|_{L^{1}(Q)}
$$

as well as

$$
\begin{aligned}
& t_{*}\left|(D a)_{i i}+(D a)_{j j}-(D a)_{i j}-(D a)_{j i}\right| \\
& =\left|\left(e_{i}-e_{j}\right) \cdot\left(a\left(z_{0}+t_{*} e_{i}\right)-a\left(z_{0}+t_{*} e_{j}\right)\right)\right| \leq c\|e(u)\|_{L^{1}(Q)},
\end{aligned}
$$


which together yield

$$
\left|D a+D a^{T}\right| \leq c\|e(u)\|_{L^{1}\left(Q_{1}\right)} .
$$

We define $w(x)=u(x)-a(x)$, so that $w\left(z_{i}\right)=0$ for $i=0, \ldots, n$.

Step 3: Definition of the exceptional set $\omega$.

In order to prove a bound on $|w|(y)$ for many points $y \in Q$ we need to ensure validity of the relation $(2.5)$ for the segments $\left[z_{i}, y\right]$. We first observe that for three-quarters of the choices of $z_{0} \in q$ one has

$$
\int_{S^{n-1}} \int_{\mathbb{R}} T\left(z_{i}, \xi, t\right) d t d \mathcal{H}^{n-1}(\xi) \leq c \mathcal{H}^{n-1}\left(J_{u}\right)
$$

for all $i$, with a constant $c$ which depends only on $n$. To see this, it suffices to integrate (2.7) over $\xi \in S^{n-1}$ and swap the order of integation. This is the third condition on $z_{0}$, and we still have at least one-quarter of $q$ at our disposal, which we shall use later to enforce a fourth condition.

For every $i=0, \ldots, n$ we define the set

$$
\omega_{(i)}=\left\{y \in Q: y=z_{i}+t \xi \text { with } T\left(z_{i}, \xi, t\right)=1\right\},
$$

which by (2.12) it has measure bounded by

$$
\left|\omega_{(i)}\right|=\int_{S^{n-1}} \int_{0}^{\infty} t^{n-1} T\left(z_{i}, \xi, t\right) d t d \mathcal{H}^{n-1}(\xi) \leq c \mathcal{H}^{n-1}\left(J_{u}\right) .
$$

We define

$$
\omega=\bigcup_{i=0}^{n} \omega_{(i)}
$$

and observe that (2.5) holds for any segment $\left[z_{i}, y\right]$ with $y \in Q \backslash \omega$ and $i \in\{0, \ldots, n\}$. Since $w$ differs from $u$ only by the affine function $a,(2.5)$ holds also for $w$.

Step 4: Estimate for $w$ on $Q \backslash \omega$.

It remains to ensure integrability of the strain along the relevant segments. We write, using $(2.5)$ on each segment $\left[y, z_{i}\right]$,

$$
\sum_{i=0}^{n}\left|\left(y-z_{i}\right) \cdot\left(w(y)-w\left(z_{i}\right)\right)\right| \leq \sum_{i=0}^{n}\left|y-z_{i}\right| \int_{\left[y, z_{i}\right]}|e(w)| d \mathcal{H}^{1} .
$$

Since $w\left(z_{i}\right)=0$, if the vectors $y-z_{i}$ were the basis vectors we would immediately obtain an estimate on $|w|(y)$. To see that the same is true for 
the given estimate, we need to show that these $n+1$ vectors are "uniformly linearly independent", in a sense we now make clear. We first observe that the convex hull $C$ of the $n+2$ points $\left\{y, z_{0}, \ldots, z_{n}\right\}$ has volume larger than $t_{*}^{n} / n$ ! (since this is the volume of the convex hull of the $\left\{z_{i}\right\}$ alone). We now claim that $C$ coincides with the union of the convex hull of the $n+1$ sets $\left\{y, z_{0}, \ldots, z_{n}\right\} \backslash\left\{z_{i}\right\}$. To see this, pick any $p \in C$. By Caratheodory's theorem, $p$ is a convex combination of $n+1$ of the points. If $y$ is one of them, we are done. Otherwise, $p \in K=\operatorname{conv}\left\{z_{0}, \ldots, z_{n}\right\}$. Let $q$ be the last point of the ray $y+[0, \infty)(p-y)$ contained in $K$. Since $q$ is on the boundary of $K$, it is a convex combination of $n$ of the $z_{i}$, therefore $p$ is a convex combination of $y$ and $n$ of the $z_{i}$, concluding the proof of the claim.

Therefore for any $y$ there are $n$ of the points $\left\{z_{0}, \ldots, z_{n}\right\}$ such that the simplex they generate has volume at least $t_{*}^{n} /(n+1)$ !. This means, that the $n$ corresponding vectors $y-z_{i}$ have the property that the determinant of the $n \times n$ matrix of which they are the columns is at least $t_{*}^{n} /(n+1) \geq 2^{-n} /(n+1)$. This $n \times n$ matrix $A$ has coefficients $a_{i, j} \in[-2,2]$ for all $i, j$, so that its comatrix is bounded by some constant $C(n)$ depending only on $n$. It follows $\left|A^{-1}\right| \leq C(n) /|\operatorname{det} A|$ and therefore

$$
\frac{|\operatorname{det} A|}{C(n)}|x| \leq|A x| \quad \text { for all } x \in \mathbb{R}^{n} .
$$

Consequently, bounding the left-hand side of (2.14) from below and using that $w\left(z_{i}\right)=0$, we obtain

$$
|w(y)| \leq c \sum_{i=0}^{n} \int_{\left[y, z_{i}\right]}|e(w)| d \mathcal{H}^{1} \quad \text { for all } y \in Q \backslash \omega
$$

and with Hölder's inequality

$$
|w|^{p}(y) \leq c \sum_{i=0}^{n} \int_{\left[y, z_{i}\right]}|e(w)|^{p} d \mathcal{H}^{1} \quad \text { for all } y \in Q \backslash \omega .
$$

It remains to integrate in $y$. To estimate the contribution of the $i$-th term in (2.15) we set $g=|e(w)|^{p} \chi_{Q}$ and change variables from $y$ to $\tilde{y}=y-z_{0} \in$ $Q_{2}=(-2,2)^{n}$,

$$
\begin{aligned}
\int_{Q \backslash \omega} \int_{\left[y, z_{i}\right]}|e(w)|^{p} d \mathcal{H}^{1} d y & \leq \int_{Q_{2}} \int_{\left[z_{0}+\tilde{y}, z_{0}+e_{i}\right]} g(x) d \mathcal{H}^{1}(x) d \tilde{y} \\
& =\int_{Q_{2}} \int_{\left[\tilde{y}, e_{i}\right]} g\left(z_{0}+t\right) d \mathcal{H}^{1}(t) d \tilde{y}
\end{aligned}
$$


We sum over $i$ and set

$$
H\left(z_{0}\right)=\sum_{i=0}^{n} \int_{Q_{2}} \int_{\left[\tilde{y}, e_{i}\right]} g\left(z_{0}+t\right) d \mathcal{H}^{1}(t) d \tilde{y},
$$

so that

$$
\int_{Q \backslash \omega}|w|^{p}(y) d y \leq c H\left(z_{0}\right) .
$$

Swapping the order of integration, we estimate as usual

$$
\int_{q} H\left(z_{0}\right) d z_{0} \leq \sum_{i=0}^{n} \int_{Q_{2}} \int_{\left[e_{i}, \tilde{y}\right]}\|g\|_{L^{1}\left(\mathbb{R}^{n}\right)} d \mathcal{H}^{1}(t) d \tilde{y} \leq c\|e(w)\|_{L^{p}\left(Q_{1}\right)}^{p}
$$

where $c$ depends only on $n$. Therefore there are many choices of $z_{0}$ (more than three-quarters) such that

$$
H\left(z_{0}\right) \leq 5 c\|e(w)\|_{L^{p}\left(Q_{1}\right)}^{p} .
$$

This is the fourth and last condition we impose on $z_{0}$. At this point we can fix $z_{0}$ such that the four conditions (2.8), (2.9), (2.12), and (2.17) are fulfilled. Recalling that $(2.11)$ gives $\|e(w)\|_{L^{p}\left(Q_{1} \backslash \omega\right)} \leq c\|e(u)\|_{L^{p}\left(Q_{1}\right)},(2.16)$ and (2.17) give

$$
\int_{Q \backslash \omega}|w|^{p}(y) d y \leq c H\left(z_{0}\right) \leq c\|e(w)\|_{L^{p}\left(Q_{1}\right)}^{p} \leq c\|e(u)\|_{L^{p}\left(Q_{1}\right)}^{p}
$$

and conclude the proof.

\section{$3 \quad$ Higher integrability}

In this Section we show that control of $e(u)$ in $L^{p}$ gives an estimate for $u-a$ in a space better than $L^{p}$. However, we only get the estimate in the optimal space $L^{p *}$ if $p=1$. The basic strategy is to use rigidity in finitely many directions $\xi$, and then to combine estimates along different directions in a way which is similar to the one used in proving the Sobolev embedding of $W^{1,1}$ into $L^{(n-1) / n}$, see Lemma 4 below. The standard argument for $u \in W^{1, p}$, based upon considering $f=|u|^{p} \in W^{1,1}$ which obeys $|D f| \leq p|u|^{p-1}|D u|$, does not apply here since we only control $u$ on part of the domain.

Proposition 3. Let $Q=(-r, r)^{n}, Q^{\prime}=(-r / 2, r / 2)^{n}, u \in S B D(Q), p \in$ $[1, \infty)$. 


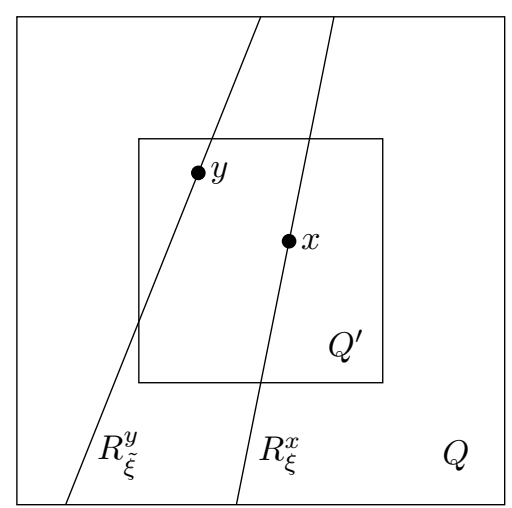

Figure 4: Sketch of the geometry in Proposition 3.

(i) There exist a set $\omega \subset Q^{\prime}$ and an affine function $a: \mathbb{R}^{n} \rightarrow \mathbb{R}^{n}$ with $e(a)=0$ such that

$$
\mathcal{L}^{n}(\omega) \leq \operatorname{cr} \mathcal{H}^{n-1}\left(J_{u}\right)
$$

and

$$
\int_{Q^{\prime} \backslash \omega}|u-a|^{n p /(n-1)} d x \leq c r^{n(p-1) /(n-1)}\left(\int_{Q}|e(u)|^{p} d x\right)^{n /(n-1)} .
$$

(ii) If additionally $p>1$ then there is $q>0$ (depending on $p$ and $n$ ) such that, for a given mollifier $\varphi_{r} \in C_{c}^{\infty}\left(B_{r / 4}\right), \varphi_{r}(x)=r^{-n} \varphi_{1}(x / r)$, the function $v=u \chi_{Q^{\prime} \backslash \omega}+a \chi_{\omega}$ obeys

$$
\int_{Q^{\prime \prime}}\left|e\left(v * \varphi_{r}\right)-e(u) * \varphi_{r}\right|^{p} d x \leq c\left(\frac{\mathcal{H}^{n-1}\left(J_{u}\right)}{r^{n-1}}\right)^{q} \int_{Q}|e(u)|^{p} d x
$$

where $Q^{\prime \prime}=(-r / 4, r / 4)^{n}$.

The constant in (i) depends only on $p$ and $n$, the one in (ii) also on $\varphi_{1}$.

Proof. By Proposition 2 applied to $Q=(-r, r)^{n}$ there exist a set $\omega_{0} \subset Q$ with $\left|\omega_{0}\right| \leq \operatorname{cr}^{n-1}\left(J_{u}\right)$ and an affine function $a$ with $e(a)=0$ such that

$$
\int_{Q \backslash \omega_{0}}|u-a|^{p} d x \leq c r^{p} \int_{Q}|e(u)|^{p} d x .
$$

We can assume without loss of generality that $a=0$ (otherwise we replace $u$ by $u-a$ ) and $r=1$ (by scaling). For $\xi \in S^{n-1}$ and $x \in Q^{\prime}$ we define the ray

$$
R_{\xi}^{x}=(x+\mathbb{R} \xi) \cap Q,
$$


see Figure 4. Then, $R_{\xi}^{x}$ is a segment and $\mathcal{H}^{1}\left(R_{\xi}^{x}\right) \geq 1$.

For any direction $\xi \in S^{n-1}$, the structure theorem [ACD97, Th. 4.5] states that for almost every $z$ for which $R_{\xi}^{z} \cap J_{u}=\emptyset$ one has, in a way which closely corresponds to (2.5) in the proof of Proposition 2,

$\xi \cdot(u(y)-u(x))=\int_{0}^{1} \xi \cdot e(u)(x+s(y-x))(y-x) d s$ for $\mathcal{H}^{1}$-a.e. $x, y \in R_{\xi}^{z}$.

For $\xi \in S^{n-1}$ we define the "shadow" of the jump set

$$
\omega_{\xi}=\left\{z \in Q^{\prime}: \text { (3.4) does not hold }\right\} \text {. }
$$

This corresponds, up to a null set, to the points $z$ such that a line through $z$ parallel to $\xi$ intersects the jump set, hence to the points in which we cannot use the estimate in direction $\xi$, and it obeys $\mathcal{H}^{n-1}\left(\Pi_{\xi} \omega_{\xi}\right) \leq \mathcal{H}^{n-1}\left(J_{u}\right)$, where $\Pi_{\xi}: \mathbb{R}^{n} \rightarrow \mathbb{R}^{n}$ denotes the orthogonal projection onto the $(n-1)$ dimensional space $\xi^{\perp}$. We remark that this set is cylindrical, in the sense that $\omega_{\xi}=\left(\omega_{\xi}+\xi \mathbb{R}\right) \cap Q^{\prime}$.

Given a direction $\xi \in S^{n-1}$ we define the exceptional set

$$
\omega_{\xi}^{*}=\omega_{\xi} \cup\left\{z \in Q^{\prime}: \mathcal{H}^{1}\left(R_{\xi}^{z} \cap \omega_{0}\right) \geq 1 / 2\right\},
$$

where $\omega_{0}$ is the set entering (3.3), which was obtained from Proposition 2. It is easy to see that $\mathcal{H}^{n-1}\left(\Pi_{\xi} \omega_{\xi}^{*}\right) \leq c \mathcal{H}^{n-1}\left(J_{u}\right)$.

For almost all $x \in Q^{\prime} \backslash \omega_{\xi}^{*},(3.4)$ gives

$$
|\xi \cdot u(x)| \leq|\xi \cdot u(y)|+\int_{R_{\xi}^{x}}|e(u)| d \mathcal{H}^{1} \quad \text { for a.e. } y \in R_{\xi}^{x}
$$

Averaging over all $y \in R_{\xi}^{x} \backslash \omega_{0}$ gives

$$
|\xi \cdot u|(x) \leq \frac{1}{\mathcal{H}^{1}\left(R_{\xi}^{x} \backslash \omega_{0}\right)} \int_{R_{\xi}^{x} \backslash \omega_{0}}|u| d \mathcal{H}^{1}+\int_{R_{\xi}^{x}}|e(u)| d \mathcal{H}^{1}
$$

and

$$
|\xi \cdot u|(x) \leq 2 \int_{x+\mathbb{R} \xi} f d \mathcal{H}^{1} \quad \text { for a.e. } x \in Q^{\prime} \backslash \omega_{\xi}^{*},
$$

where

$$
f=|e(u)| \chi_{Q}+|u| \chi_{Q \backslash \omega_{0}} .
$$

We observe that by (3.3) the function $f$ obeys

$$
\|f\|_{L^{p}\left(\mathbb{R}^{n}\right)} \leq c\|e(u)\|_{L^{p}(Q)} .
$$


We choose a set $S$ containing $n^{2}$ vectors of $S^{n-1}$ with the property that any $n$ of them are linearly independent. To show that they exist it suffices to choose them iteratively, at any step only a null set needs to be avoided. These vectors are universal and will influence the constants in the estimate. At variance with Proposition 2 we shall use here only finitely many directions.

For any set $V \subset S$ of $n$ vectors (which are automatically linearly independent) one has

$$
|u|(x) \leq c_{V} \sum_{\xi \in V}|u \cdot \xi|(x),
$$

with a constant which depends only on $V$. We define

$$
\omega=\bigcup_{\xi \in S} \omega_{\xi}^{*}
$$

which obeys $\omega \subset Q^{\prime},|\omega| \leq c \mathcal{H}^{n-1}\left(J_{u}\right)$ and therefore (3.1). Recalling (3.5),

$$
|u|(x) \leq c_{V} \sum_{\xi \in V} \int_{x+\mathbb{R} \xi} f d \mathcal{H}^{1} \quad \text { for a.e. } x \in Q^{\prime} \backslash \omega
$$

and by Hölder's inequality

$$
|u|^{p}(x) \leq c_{V} \sum_{\xi \in V} \int_{x+\mathbb{R} \xi} f^{p} d \mathcal{H}^{1} \quad \text { for a.e. } x \in Q^{\prime} \backslash \omega .
$$

At this point we use the estimate (3.7) with several different sets of vectors. Let $V_{1}, \ldots, V_{n}$ be $n$ disjoint sets of $n$ vectors in $S$. Then (3.7) holds for each of them. We multiply these $n$ equations and obtain for almost every $x \in Q^{\prime} \backslash \omega$

$$
|u|^{n p}(x) \leq \prod_{j=1}^{n} c_{V_{j}} \sum_{\xi \in V_{j}} \int_{x+\mathbb{R} \xi} f^{p} d \mathcal{H}^{1} \quad \text { for a.e. } x \in Q^{\prime} \backslash \omega .
$$

Since there are finitely many choices of $V \subset S$ the constant is universal. We swap the sum with the product and obtain

$$
|u|^{n p}(x) \leq c_{S} \sum_{W \subset S, \# W=n} \prod_{\xi \in W} \int_{x+\mathbb{R} \xi} f^{p} d \mathcal{H}^{1} \quad \text { for a.e. } x \in Q^{\prime} \backslash \omega,
$$

where by the definition of $S$ the vectors in any of the sets $W=\left\{\xi_{1}, \ldots, \xi_{n}\right\} \subset$ $S$ are linearly independent (the relevant sets $W$ are those which contain one vector from each of the $V_{j}$ ). By Lemma 4 (see below) applied to each term in the sum and $f^{p}$ we obtain

$$
\left\|u \chi_{Q^{\prime} \backslash \omega}\right\|_{L^{n p /(n-1)}} \leq c\|f\|_{L^{p}} .
$$


Recalling (3.6) the proof of (3.2) and therefore of part (i) is concluded.

We now turn to assertion (ii) concerning the function $v=u \chi_{Q^{\prime} \backslash \omega}$. We define, with $\varphi=\varphi_{1} \in C_{c}^{\infty}\left(B_{1 / 4}\right)$ as in the statement, a function $h \in$ $C^{\infty}\left(Q^{\prime \prime}, \mathbb{R}_{\mathrm{sym}}^{n \times n}\right)$ by

$$
h=e(v * \varphi)-e(u) * \varphi .
$$

To conclude the proof it suffices to bound $\xi \cdot h \xi$ in $L^{p}\left(Q^{\prime \prime}\right)$ for a finite set of $\xi$. For example, all $\xi \in T=\left\{e_{i}\right\}_{i=1, \ldots, n} \cup\left\{\left(e_{i}+e_{j}\right) / \sqrt{2}\right\}_{i, j=1 \ldots n}$ will do, since $A \mapsto \sum_{\xi \in T}|\xi \cdot A \xi|$ is a norm on $\mathbb{R}_{\mathrm{sym}}^{n \times n}$.

We fix $\xi \in S^{n-1}$ and define, for $\eta \in(0,1 / 2)$ chosen below,

$$
\omega_{\xi}^{* *}=\omega_{\xi} \cup\left\{z \in Q^{\prime}: \mathcal{H}^{1}\left(R_{\xi}^{z} \cap\left(\omega \cup \omega_{0}\right)\right) \geq \eta\right\},
$$

which obeys $\omega_{\xi}^{*} \subset \omega_{\xi}^{* *}$ and

$$
\left|\omega_{\xi}^{* *}\right| \leq c \frac{\mathcal{H}^{n-1}\left(J_{u}\right)}{\eta}
$$

We pick $z \in Q^{\prime} \backslash \omega_{\xi}^{* *}$. Then $z \notin \omega_{\xi}^{*}$ and, since the latter set is cylindrical, $R_{\xi}^{z} \cap \omega_{\xi}^{*}=\emptyset$. Therefore (3.5) gives

$$
|\xi \cdot u|(x) \leq 2 \int_{R_{\xi}^{z}} f d \mathcal{H}^{1} \quad \text { for a.e. } x \in Q^{\prime} \cap R_{\xi}^{z},
$$

and, with Hölder's inequality,

$$
|\xi \cdot u|^{p}(x) \leq c \int_{R_{\xi}^{z}} f^{p} d \mathcal{H}^{1} \text { for a.e. } x \in Q^{\prime} \cap R_{\xi}^{z} .
$$

Integration over all $x \in \omega \cap R_{\xi}^{z} \subset Q^{\prime} \cap R_{\xi}^{z}$ gives, since $\mathcal{H}^{1}\left(\omega \cap R_{\xi}^{z}\right)<\eta$,

$$
\int_{\omega \cap R_{\xi}^{z}}|\xi \cdot u|^{p} d \mathcal{H}^{1} \leq c \eta \int_{R_{\xi}^{z}} f^{p} d \mathcal{H}^{1} .
$$

Integrating then over $z \in Q^{\prime} \backslash \omega_{\xi}^{* *}$ and using (3.6) yields

$$
\int_{\omega \backslash \omega_{\xi}^{* *}}|\xi \cdot u|^{p} d x \leq c \eta \int_{Q} f^{p} d x \leq c \eta\|e(u)\|_{L^{p}(Q)}^{p} .
$$

For the same $\xi$ we define the longitudinal component $w^{(\xi)}: \mathbb{R}^{n} \rightarrow \mathbb{R}$ by

$$
w^{(\xi)}=\xi \cdot u \chi_{Q^{\prime} \backslash \omega_{\xi}^{* *}}
$$


We now show that for any $z \in Q^{\prime}$ the function $t \mapsto w^{(\xi)}(z+t \xi)$ is in $W^{1,1}(I)$, with $I=\left\{t \in \mathbb{R}: z+t \xi \in Q^{\prime}\right\}$, and

$$
\frac{d}{d t} w^{(\xi)}(z+t \xi)=\left[\xi \cdot e(u) \xi \chi_{Q^{\prime} \backslash \omega_{\xi}^{* *}}\right](z+t \xi) .
$$

To see this, we observe that $\omega_{\xi}^{* *}$ is cylindrical, in the sense that $\omega_{\xi}^{* *}=\left(\omega_{\xi}^{* *}+\right.$ $\xi \mathbb{R}) \cap Q^{\prime}$. If $z \in \omega_{\xi}^{* *}$ then $w^{(\xi)}(z+t \xi)=0$ for all $t$ and (3.12) holds. If instead $z \notin \omega_{\xi}^{* *}$ then $\chi_{Q^{\prime} \backslash \omega_{\xi}^{* *}}(z+t \xi)=1$ for all $t \in I$, so that $w^{(\xi)}=\xi \cdot u$. Since $z \notin \omega_{\xi}(3.4)$ holds, and this implies $t \mapsto \xi \cdot u(z+t \xi) \in W^{1,1}(I)$ with derivative $\xi \cdot e(u) \xi(z+t \xi)$. This proves (3.12).

In particular, $D_{\xi} w^{(\xi)}=\xi \cdot e(u) \xi \chi_{Q^{\prime} \backslash \omega_{\xi}^{* *}}$ and therefore in $Q^{\prime \prime}$ we have, recalling (3.9),

$$
\begin{aligned}
\xi \cdot h \xi & =D_{\xi}(\xi \cdot v * \varphi)-\xi \cdot e(u) \xi * \varphi \\
& =D_{\xi}\left(\left(\xi \cdot v-w^{(\xi)}\right) * \varphi\right)+D_{\xi}\left(w^{(\xi)} * \varphi\right)-\xi \cdot e(u) \xi * \varphi \\
& =D_{\xi}\left(\left(\xi \cdot v-w^{(\xi)}\right) * \varphi\right)-\xi \cdot\left(e(u) \chi_{\omega_{\xi}^{* *}}\right) \xi * \varphi
\end{aligned}
$$

We estimate the two terms in (3.13) separately in $L^{p}\left(Q^{\prime \prime}\right)$.

In the first term we use $|D \varphi| \leq c$ to obtain

$$
\int_{Q^{\prime \prime}}\left|D_{\xi}\left(\left(\xi \cdot v-w^{(\xi)}\right) * \varphi\right)\right|^{p} d x \leq c \int_{Q^{\prime}}\left|\xi \cdot v-w^{(\xi)}\right|^{p} d x
$$

Since $w^{(\xi)}=\xi \cdot v=\xi \cdot u$ on $Q^{\prime} \backslash\left(\omega \cup \omega_{\xi}^{* *}\right), w^{(\xi)}=0$ on $\omega_{\xi}^{* *}$, and $\xi \cdot v=0$ on $\omega$,

$$
\int_{Q^{\prime}}\left|\xi \cdot v-w^{(\xi)}\right|^{p} d x=\int_{\omega \backslash \omega_{\xi}^{* *}}\left|w^{(\xi)}\right|^{p} d x+\int_{\omega_{\xi}^{* *} \backslash \omega}|\xi \cdot v|^{p} d x .
$$

The last term, using Hölder's inequality, (3.10) and (3.2), is controlled by

$$
\begin{aligned}
\int_{\omega_{\xi}^{* *} \backslash \omega}|\xi \cdot v|^{p} & \leq\left|\omega_{\xi}^{* *}\right|^{1 / n}\left(\int_{Q^{\prime} \backslash \omega}|v|^{n p /(n-1)}\right)^{(n-1) / n} \\
& \leq c\left(\frac{\mathcal{H}^{n-1}\left(J_{u}\right)}{\eta}\right)^{1 / n}\|e(u)\|_{L^{p}(Q)}^{p}
\end{aligned}
$$

Instead, the first one is controlled by (3.11), which gives

$$
\int_{\omega \backslash \omega_{\xi}^{* *}}\left|w^{(\xi)}\right|^{p} d x \leq c \eta\|e(u)\|_{L^{p}(Q)}^{p} .
$$


In the second term in (3.13) we estimate

$$
\begin{aligned}
\left\|e(u) \chi_{\omega_{\xi}^{* *}} * \varphi\right\|_{L^{p}\left(Q^{\prime \prime}\right)} & \leq\left\|e(u) \chi_{\omega_{\xi}^{* *}}\right\|_{L^{1}\left(Q^{\prime}\right)}\|\varphi\|_{L^{p}\left(\mathbb{R}^{n}\right)} \\
& \leq\left\|\chi_{\omega_{\xi}^{* * *}}\right\|_{L^{p^{\prime}}\left(Q^{\prime}\right)}\|e(u)\|_{L^{p}\left(Q^{\prime}\right)}\|\varphi\|_{L^{p}\left(\mathbb{R}^{n}\right)} \\
& \leq c\left(\frac{\mathcal{H}^{n-1}\left(J_{u}\right)}{\eta}\right)^{1-1 / p}\|e(u)\|_{L^{p}\left(Q^{\prime}\right)},
\end{aligned}
$$

where we used Hölder's inequality with $p^{\prime}$ defined by $1 / p+1 / p^{\prime}=1$ and (3.10).

Combining these estimates with (3.13) leads to

$$
\|\xi \cdot h \xi\|_{L^{p}\left(Q^{\prime \prime}\right)} \leq c\left[\left(\frac{\mathcal{H}^{n-1}\left(J_{u}\right)}{\eta}\right)^{1 /(n p)}+\eta^{1 / p}+\left(\frac{\mathcal{H}^{n-1}\left(J_{u}\right)}{\eta}\right)^{1-1 / p}\right]\|e(u)\|_{L^{p}(Q)} .
$$

If $1 /(n p) \leq 1-1 / p$ we choose $\eta=\left(\mathcal{H}^{n-1}\left(J_{u}\right)\right)^{1 /(n+1)}$ and obtain

$$
\|h\|_{L^{p}\left(Q^{\prime \prime}\right)} \leq c\left(\mathcal{H}^{n-1}\left(J_{u}\right)\right)^{1 /(p(n+1))}\|e(u)\|_{L^{p}(Q)} .
$$

If instead $1 /(n p)>1-1 / p$ we choose $\eta=\left(\mathcal{H}^{n-1}\left(J_{u}\right)\right)^{(p-1) / p}$ and obtain

$$
\|h\|_{L^{p}\left(Q^{\prime \prime}\right)} \leq c\left(\mathcal{H}^{n-1}\left(J_{u}\right)\right)^{(p-1) / p^{2}}\|e(u)\|_{L^{p}(Q)} .
$$

Lemma 4. Let $V \subset S^{n-1}$ be a set of $n$ linearly independent vectors, $u, f \in$ $L^{1}\left(\mathbb{R}^{n}\right)$ obey

$$
|u|^{n}(x) \leq \prod_{i=1}^{n} \int_{x+\mathbb{R} \xi_{i}} f d \mathcal{H}^{1}
$$

for almost every $x \in \mathbb{R}^{n}$. Then $u \in L^{n /(n-1)}\left(\mathbb{R}^{n}\right)$ and

$$
\|u\|_{L^{n /(n-1)\left(\mathbb{R}^{n}\right)}} \leq c\|f\|_{L^{1}\left(\mathbb{R}^{n}\right)} .
$$

Proof. This is part of the standard proof of Sobolev inequality, as discussed for example in [Eva98, Eq. (11-13), pages 263-264]. We remark that this part of the proof only deals with Fubini and iterated integration and only requires $f \in L^{1}\left(\mathbb{R}^{n}\right)$.

\section{Estimate up to the boundary}

In this section we refine the argument from the previous one in several directions. We obtain a better estimate of the volume of the exceptional set, with 
the isoperimetric scaling, we obtain an estimate up to the boundary, and an estimate for the trace. A covering argument will then lead to the proof of Theorem 1.

One important ingredient in proving a better estimate for the volume of the exceptional set is the following isoperimetric estimate, which bounds the volume of a set from the area of its codimension-1 projections.

Lemma 5. Let $n \geq 2, \Omega \subset \mathbb{R}^{n}$ be measurable and bounded, let $\xi_{1} \ldots, \xi_{n} \in$ $S^{n-1}$ be linearly independent vectors. Then

$$
\mathcal{L}^{n}(\Omega) \leq c \prod_{i=1}^{n}\left(\mathcal{H}^{n-1}\left(\prod_{\xi_{i}} \Omega\right)\right)^{1 /(n-1)} .
$$

The constant $c$ depends only on the vectors $\xi$. Here $\prod_{\xi}: \mathbb{R}^{n} \rightarrow \mathbb{R}^{n}$ denotes the projection onto the $n-1$-dimensional space $\xi^{\perp}, \Pi_{\xi}=\mathrm{Id}-\xi \otimes \xi /|\xi|^{2}$.

Proof. After a linear transformation we can assume $\xi_{i}=e_{i}$. We shall prove that

$$
\mathcal{L}^{n}(\Omega) \leq \prod_{i=1}^{n}\left(\mathcal{H}^{n-1}\left(\Pi_{e_{i}} \Omega\right)\right)^{1 /(n-1)} .
$$

We write $\omega_{i}=\Pi_{e_{i}} \Omega$. If $n=2$ one has

$$
\chi_{\Omega}\left(x_{1}, x_{2}\right) \leq \chi_{\omega_{2}}\left(x_{1}, 0\right) \chi_{\omega_{1}}\left(0, x_{2}\right)
$$

and integrating with Fubini's theorem gives

$$
\mathcal{L}^{2}(\Omega)=\int_{\mathbb{R}^{2}} \chi_{\Omega} d x \leq \mathcal{H}^{1}\left(\omega_{1}\right) \mathcal{H}^{1}\left(\omega_{2}\right)
$$

To prove (4.2) in higher dimension we proceed by induction on $n$. We define for $t \in \mathbb{R}$

$$
\Omega_{t}=\Omega \cap\left\{x_{1}=t\right\} \quad \text { and } \quad h(t)=\mathcal{H}^{n-1}\left(\Omega_{t}\right) .
$$

From $\Pi_{e_{1}} \Omega_{t} \subset \omega_{1}$ one obtains $h(t) \leq \mathcal{H}^{n-1}\left(\omega_{1}\right)$ for all $t$. Projecting in the other directions we obtain

$$
\Pi_{e_{i}} \Omega_{t}=\omega_{i} \cap\left\{x_{1}=t\right\} \quad \text { for } i=2, \ldots, n \text {. }
$$

By the inductive assumption applied to the $n-1$ dimensional space $\left\{x_{1}=t\right\}$ we obtain

$$
h(t) \leq \prod_{i=2}^{n}\left(\mathcal{H}^{n-2}\left(\omega_{i} \cap\left\{x_{1}=t\right\}\right)\right)^{1 /(n-2)} .
$$


From $h(t) \leq \min \{a, b\}$ it follows that $h(t) \leq a^{1 /(n-1)} b^{(n-2) /(n-1)}$, and therefore

$$
h(t) \leq\left(\mathcal{H}^{n-1}\left(\omega_{1}\right)\right)^{1 /(n-1)} \prod_{i=2}^{n}\left(\mathcal{H}^{n-2}\left(\omega_{i} \cap\left\{x_{1}=t\right\}\right)\right)^{1 /(n-1)} .
$$

With Hölder's inequality we obtain

$$
\int_{\mathbb{R}} h(t) d t \leq\left(\mathcal{H}^{n-1}\left(\omega_{1}\right)\right)^{1 /(n-1)} \prod_{i=2}^{n}\left(\int_{\mathbb{R}} \mathcal{H}^{n-2}\left(\omega_{i} \cap\left\{x_{1}=t\right\}\right) d t\right)^{1 /(n-1)}
$$

and therefore, by Fubini's theorem,

$$
\mathcal{L}^{n}(\Omega)=\int_{\mathbb{R}} h(t) d t \leq \prod_{i=1}^{n}\left(\mathcal{H}^{n-1}\left(\omega_{i}\right)\right)^{1 /(n-1)} .
$$

This concludes the proof of (4.2) and therefore that of the Lemma.

We now present the main local estimate. The proof is similar to that of Proposition 3, but refined in various directions. As in Proposition 3 we estimate $u(x)$ by controlling $|\xi \cdot u|(x)$ for a set of $n$ distinct, uniformly linearly independent vectors $\xi$. In this case the admissible $\xi$ are all chosen in the small set $\tilde{S}$, corresponding to a uniform "interior cone condition" for the relevant part of the boundary, so that they all point "outside", permitting to obtain the estimate on $u$ up to the boundary and, therefore, on the trace. Using $n^{2}$ vectors instead of $n$ gives higher integrability, as in Proposition 3 . The improved estimate for the size of the exceptional set $\omega$ is obtained by noticing that a point $x$ needs to be included in $\omega$ only if there is no choice of a set of directions $\xi$ which permits to estimate $u(x)$. Therefore we use first a larger (but still finite) set $S$ of vectors, containing $n^{2}+n-1$ elements and still contained in the cone; each subset $Z \subset S$ containing $n^{2}$ vectors leads to an estimate in $L^{n p /(p-1)}$ outside a suitable ( $Z$-dependent) exceptional set. The set $\omega$ is then the intersection of all these exceptional sets, and its volume can be estimated with Lemma 5 .

Proposition 6. For $r>0, \varphi \in \operatorname{Lip}\left((-r, r)^{n-1},[r, \infty)\right)$ with inf $\varphi=r$, and $A: \mathbb{R}^{n} \rightarrow \mathbb{R}^{n}$ an affine isometry we define the open sets

$$
\Omega=A\left\{\left(x^{\prime}, x_{n}\right) \in(-r, r)^{n-1} \times \mathbb{R}:-r<x_{n}<\varphi\left(x^{\prime}\right)\right\}
$$

and

$$
\Omega^{\text {int }}=A\left\{\left(x^{\prime}, x_{n}\right) \in(-r / 2, r / 2)^{n-1} \times \mathbb{R}: 0<x_{n}<\varphi\left(x^{\prime}\right)\right\} .
$$

Let $u \in S B D(\Omega)$. Then there is an affine function $a: \mathbb{R}^{n} \rightarrow \mathbb{R}^{n}$ with $e(a)=0$ such that: 
(i) There is a set $\omega \subset \Omega^{\text {int }}$ such that

$$
\mathcal{L}^{n}(\omega) \leq c_{L}\left(\mathcal{H}^{n-1}\left(J_{u}\right)\right)^{n /(n-1)}
$$

and

$$
\|u-a\|_{L^{n p /(n-1)}\left(\Omega^{\mathrm{int}} \backslash \omega\right)} \leq c_{L} r^{1-1 / p}\|e(u)\|_{L^{p}(\Omega)} .
$$

(ii) There is a set $\omega_{\Gamma} \subset \Gamma=A\left\{\left(x^{\prime}, \varphi\left(x^{\prime}\right)\right): x^{\prime} \in(-r / 2, r / 2)^{n-1}\right\}$ such that

$$
\mathcal{H}^{n-1}\left(\omega_{\Gamma}\right) \leq c_{L} \mathcal{H}^{n-1}\left(J_{u}\right)
$$

and the trace $T u$ of $u$ on $\Gamma$ obeys

$$
\|T u-a\|_{L^{p}\left(\Gamma \backslash \omega_{\Gamma}\right)} \leq c_{L}\|e(u)\|_{L^{p}(\Omega)} .
$$

The constant $c_{L}$ depends only on $p, n$ and the Lipschitz constant $L$ of $\varphi$.

By affine isometry we mean a map $A: \mathbb{R}^{n} \rightarrow \mathbb{R}^{n}$ such that $A(x)=b+Q x$, with $b \in \mathbb{R}^{n}$ and $Q \in O(n)$. The geometry is illustrated in Figure 5 .

Proof. The first part of the proof is similar to the proof of Proposition 3. Replacing $u$ by $\tilde{u}(x)=A^{-1} u(r A x)$ we can assume $r=1$ and $A(x)=x$. By Proposition 2 applied to the cube $Q=(-1,1)^{n}$ there are a set $\omega_{0} \subset Q$ and an affine function $a$ with $e(a)=0$ such that $\left|\omega_{0}\right| \leq c \mathcal{H}^{n-1}\left(J_{u}\right)$ and

$$
\int_{Q \backslash \omega_{0}}|u-a|^{p} d x \leq c \int_{\Omega}|e(u)|^{p} d x .
$$

We can assume without loss of generality $a=0$, otherwise we replace $u$ by $u-a$. We let

$$
\tilde{S}=\left\{\xi \in S^{n-1}:\left|\xi-e_{n}\right|<\frac{1}{2 \sqrt{1+L^{2}}}\right\}
$$

Then the following holds: (i) the outer normal $\nu$ to $\partial \Omega$ in a point $x \in \Gamma$ obeys $\xi \cdot e_{n} \geq 1 / \sqrt{1+L^{2}}$ and therefore $\xi \cdot \nu \geq 1 /\left(2 \sqrt{1+L^{2}}\right)$; (ii) for any $\xi \in \tilde{S}$ and any $x \in \overline{\Omega^{\text {int }}}$ the line $x+\mathbb{R} \xi$ intersects the surfaces $\left\{\left(x^{\prime}, x_{n}\right): x_{n}=\right.$ $\left.\varphi\left(x^{\prime}\right), x^{\prime} \in(-1,1)^{n-1}\right\}$ and $(-1,1)^{n-1} \times\{-1\}$ at exactly one point each.

For $\xi \in \tilde{S}$ and $x \in \overline{\Omega^{\text {int }}}$ we define the ray

$$
R_{\xi}^{x}=(x+\mathbb{R} \xi) \cap \Omega,
$$

see Figure 5. The definition of $\tilde{S}$ ensures that $R_{\xi}^{x}$ is a segment and that $\mathcal{H}^{1}\left(R_{\xi}^{x} \cap Q\right) \geq 2$. 


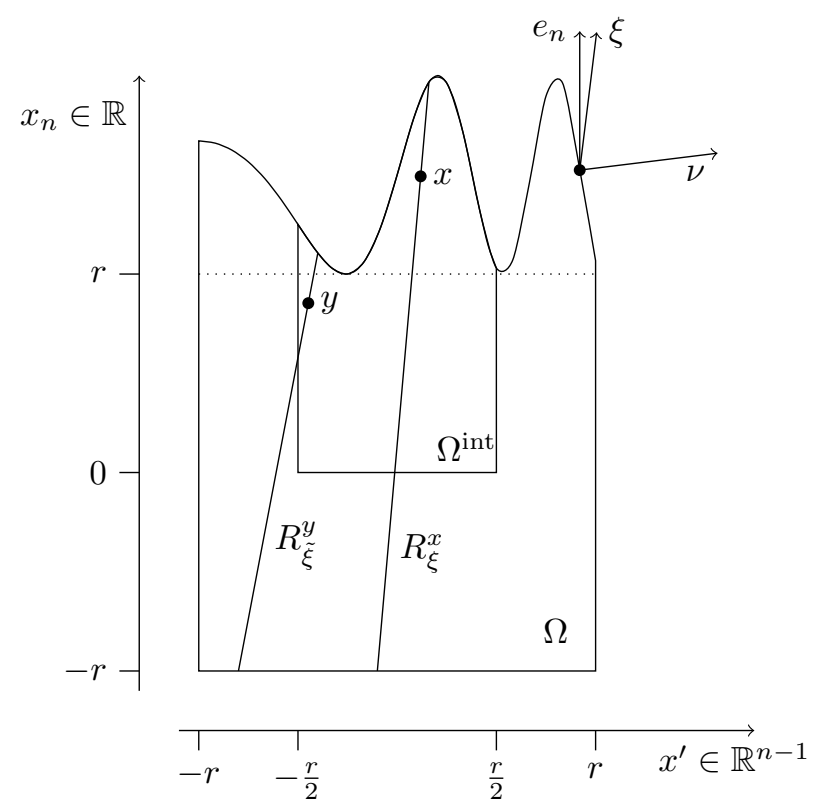

Figure 5: Sketch of the construction in Proposition 6.

For any direction $\xi \in \tilde{S}$, one has just like in (3.4) that

$$
\xi \cdot(u(y)-u(x))=\int_{0}^{1} \xi \cdot e(u)(x+s(y-x))(y-x) d s \text { for a.e. } x, y \in R_{\xi}^{z}
$$

holds for almost every $z$ for which $R_{\xi}^{z} \cap J_{u}=\emptyset$. As above we define for $\xi \in \tilde{S}$ the "shadow" of the jump set

$$
\omega_{\xi}=\left\{z \in \Omega^{\text {int }}:(4.6) \text { does not hold }\right\}
$$

and the exceptional set

$$
\omega_{\xi}^{*}=\omega_{\xi} \cup\left\{x \in \Omega^{\mathrm{int}}: \mathcal{H}^{1}\left(R_{\xi}^{x} \cap \omega_{0}\right) \geq 1\right\},
$$

which obey $\mathcal{H}^{n-1}\left(\Pi_{\xi} \omega_{\xi}\right) \leq c \mathcal{H}^{n-1}\left(J_{u}\right)$ and $\mathcal{H}^{n-1}\left(\Pi_{\xi} \omega_{\xi}^{*}\right) \leq c \mathcal{H}^{n-1}\left(J_{u}\right)$.

The same argument as in Proposition, averaging over all $y \in Q \cap R_{\xi}^{x} \backslash \omega_{0}$, 3 leads to

$$
|\xi \cdot u|(x) \leq \int_{R_{\xi}^{x}}|e(u)| d \mathcal{H}^{1}+\frac{1}{\mathcal{H}^{1}\left(Q \cap R_{\xi}^{x} \backslash \omega_{0}\right)} \int_{Q \cap R_{\xi}^{x} \backslash \omega_{0}}|u| d \mathcal{H}^{1}
$$

for a.e. $x \in \Omega^{\text {int }} \backslash \omega_{\xi}^{*}$. Since $\mathcal{H}^{1}\left(Q \cap R_{\xi}^{x} \backslash \omega\right) \geq 1$ we obtain

$$
|\xi \cdot u|^{p}(x) \leq c \int_{R_{\xi}^{x}} f^{p} d \mathcal{H}^{1} \quad \text { for a.e. } x \in \Omega^{\text {int }} \backslash \omega_{\xi}^{*},
$$


where $f=|e(u)| \chi_{\Omega}+|u| \chi_{Q \backslash \omega_{0}}$.

We let $S$ be a subset of $n^{2}+n-1$ vectors in $\tilde{S}$ with the property that any $n$ of them are linearly independent. The choice of $S$ depends only on $L$ and will influence the constants in the statement. For any set $V \subset S$ of $n$ vectors we have

$$
|u|^{p}(x) \leq c_{V} \sum_{\xi \in V}|u \cdot \xi|^{p}(x) \leq c_{V} \sum_{\xi \in V} \int_{R_{\xi}^{x}} f^{p} d \mathcal{H}^{1} .
$$

For a set $Z \subset S$ of $n^{2}$ vectors we define

$$
\omega_{Z}=\bigcup_{\xi \in Z} \omega_{\xi}^{*} .
$$

The same argument as in Proposition 3 leads to

$$
\left\|u \chi_{\Omega^{\text {int }} \backslash \omega_{Z}}\right\|_{L^{n p /(n-1)}\left(\mathbb{R}^{n}\right)} \leq c_{Z}\|f\|_{L^{p}(\Omega)} .
$$

The same holds for any possible choice of $Z$. We set

$$
\omega=\bigcap_{Z \subset S, \# Z=n^{2}} \omega_{Z}=\bigcap_{Z \subset S, \# Z=n^{2}} \bigcup_{\xi \in Z} \omega_{\xi}^{*} .
$$

Since there are finitely many possible choices of $Z$, we conclude that $\left\|u \chi_{\Omega^{\text {int }} \backslash \omega}\right\|_{L^{n p /(n-1)}\left(\mathbb{R}^{n}\right)} \leq \sum_{Z} c_{Z}\|f\|_{L^{p}(\Omega)} \leq c\|f\|_{L^{p}(\Omega)}$, which concludes the proof of (4.4).

The set $\omega$ is the set of points such that for any choice of $Z$ they lie in at least one of the $\omega_{\xi}^{*}$. Since $S$ has $n^{2}+n-1$ elements, if a point belongs to less than $n$ of the $\omega_{\xi}^{*}, \xi \in S$, then there is a choice of $Z$ such that it does not belong to $\cup_{\xi \in Z} \omega_{\xi}^{*}$. We conclude that any point in $\omega$ belongs to at least $n$ of the $\omega_{\xi}^{*}, \xi \in S$, therefore

$$
\omega \subset \bigcup_{W \subset S, \# W=n} \bigcap_{\xi \in W} \omega_{\xi}^{*}=\bigcup_{W \subset S, \# W=n} \omega^{W}, \quad \omega^{W}=\bigcap_{\xi \in W} \omega_{\xi}^{*} .
$$

For all $\xi \in S$ we have $\mathcal{H}^{n-1}\left(\Pi_{\xi} \omega_{\xi}^{*}\right) \leq c \mathcal{H}^{n-1}\left(J_{u}\right)$. For any choice of $W$, since the $n$ vectors are independent Lemma 5 implies $\left|\omega^{W}\right| \leq c \mathcal{H}^{n-1}\left(J_{u}\right)^{n /(n-1)}$. Since there are finitely many choices of $W$,

$$
|\omega| \leq \sum_{W}\left|\omega^{W}\right| \leq c \mathcal{H}^{n-1}\left(J_{u}\right)^{n /(n-1)} .
$$

This concludes the proof of (i). 
We finally turn to the estimate for the trace. We denote by $\Gamma=$ $\left\{\left(x^{\prime}, \varphi\left(x^{\prime}\right)\right): x^{\prime} \in(-1 / 2,1 / 2)^{n-1}\right\}$ the part of the graph of $\varphi$ which is contained in $\partial \Omega^{\text {int }}$, and define for $\xi \in S$

$$
\omega_{\xi}^{\Gamma}=\left\{x \in \Gamma: \mathcal{H}^{1}\left(R_{\xi}^{x} \cap \omega_{\xi}^{*}\right)>0\right\},
$$

which obeys $\prod_{\xi} \omega_{\xi}^{\Gamma} \subset \Pi_{\xi} \omega_{\xi}^{*}$ and therefore $\mathcal{H}^{n-1}\left(\omega_{\xi}^{\Gamma}\right) \leq c \mathcal{H}^{n-1}\left(J_{u}\right)$. We denote by $T u: \Gamma \rightarrow \mathbb{R}^{n}$ the trace of $u$ on $\Gamma$. For any $x \in \Gamma \backslash \omega_{\xi}^{\Gamma}$ we have

$$
|\xi \cdot u|^{p}(x-t \xi) \leq c \int_{R_{\xi}^{x}} f^{p} d \mathcal{H}^{1} \text { for a.e. } t \in(0,1 / 2)
$$

which implies, by the property of traces,

$$
|\xi \cdot T u|^{p}(x) \leq c \int_{R_{\xi}^{x}} f^{p} d \mathcal{H}^{1} \quad \text { for } \mathcal{H}^{n-1} \text {-a.e. } x \in \Gamma \backslash \omega_{\xi}^{\Gamma} .
$$

Let now $V \subset S$ be a set of $n$ vectors, which are automatically linearly independent, and set $\omega_{\Gamma}=\cup_{\xi \in V} \omega_{\xi}^{\Gamma}$. Then integrating over $x \in \Gamma \backslash \omega^{\Gamma}$ gives

$$
\int_{\Gamma \backslash \omega_{\Gamma}}|T u|^{p} d \mathcal{H}^{n-1} \leq c_{V} \sum_{\xi \in V} \int_{\Gamma \backslash \omega_{\xi}^{\Gamma}}|\xi \cdot T u|^{p} d \mathcal{H}^{n-1} \leq c\|e(u)\|_{L^{p}(\Omega)}^{p},
$$

which is the required estimate.

Proof of Theorem 1 . We choose finitely many sets $\Omega_{1}, \ldots, \Omega_{M}, \Omega_{1}^{\text {int }}, \ldots, \Omega_{M}^{\text {int }}$ of the type entering Proposition 6 (up to rotations and translations) such that $\partial \Omega$ is covered by the $\overline{\Omega_{i}^{\text {int }}}$. Then we choose finitely many cubes $\Omega_{M+1}^{\text {int }}, \ldots, \Omega_{K}^{\text {int }}$ which cover $\Omega \backslash \cup \overline{\Omega_{i}^{\text {int }}}$ such that the double cubes $\Omega_{M+1}, \ldots, \Omega_{K}$ are contained in $\Omega$. On each of them we can apply Proposition 6 (using $\varphi\left(x^{\prime}\right)=r$ for the cubes). We can assume that $J_{u}$ is sufficiently small that

$$
c_{L}\left(\mathcal{H}^{n-1}\left(J_{u}\right)\right)^{n /(n-1)} \leq \frac{1}{3} \min \left\{\left|\Omega_{i}^{\mathrm{int}} \cap \Omega_{j}^{\mathrm{int}}\right|: \Omega_{i}^{\mathrm{int}} \cap \Omega_{j}^{\mathrm{int}} \neq \emptyset\right\},
$$

otherwise $\omega=\Omega$ will do (here $c_{L}$ is the constant in Proposition 6, which depends only on $p, n$ and the Lipschitz constant of $\Omega$ ). We obtain finitely many exceptional sets $\omega_{1}, \ldots, \omega_{K}$ and $\omega_{\Gamma}^{1}, \ldots, \omega_{\Gamma}^{M}$ (for the inner cubes we do not need the boundary estimate) and finitely many affine functions $a_{1}, \ldots, a_{K}$. These functions are in a finite-dimensional space, and since $\Omega$ is connected

$$
\sum_{i, j}\left\|a_{i}-a_{j}\right\|_{L^{1}\left(\Omega_{i}^{\text {int }} \cap \Omega_{j}^{\text {int }}\right)}
$$


is a seminorm on the space of the $K$-uples of affine functions $\left(a_{1}, \ldots, a_{K}\right)$, which vanishes on the subspace $\left\{a_{1}=\cdots=a_{K}\right\}$. By the assumption on $J_{u}$ also

$$
\sum_{i, j}\left\|a_{i}-a_{j}\right\|_{L^{1}\left(\Omega_{i}^{\text {int }} \cap \Omega_{j}^{\text {int }} \backslash\left(\omega_{i} \cup \omega_{j}\right)\right)}
$$

is a seminorm. Therefore, there exists $c>0$ such that

$$
\inf _{a \text { affine }} \sup _{i}\left\|a_{i}-a\right\|_{L^{1}\left(\Omega_{i}\right)} \leq c \sum_{i, j}\left\|a_{i}-a_{j}\right\|_{L^{1}\left(\Omega_{i}^{\mathrm{int}} \cap \Omega_{j}^{\mathrm{int}} \backslash\left(\omega_{i} \cup \omega_{j}\right)\right)}
$$

and we can choose a unique affine function. The constant depends only on $\Omega$ because for any $\alpha$ there is $c_{\alpha}>0$ such that

$$
\|a\|_{L^{1}(\Omega)} \leq c_{\alpha}\|a\|_{L^{1}(E)} \text { for all } a \text { affine, } E \subset \Omega \text { with }|E|>\alpha .
$$

Choosing $\alpha$ as the minimum of the nonzero $\left|\Omega_{i}^{\text {int }} \cap \Omega_{j}^{\text {int }}\right| / 3$, then for the same indices $\left|\Omega_{i}^{\text {int }} \cap \Omega_{j}^{\text {int }} \backslash\left(\omega_{i} \cup \omega_{j}\right)\right| \geq\left|\Omega_{i}^{\text {int }} \cap \Omega_{j}^{\text {int }}\right|-\left|\omega_{i}\right|-\left|\omega_{j}\right| \geq \alpha$.

\section{References}

[ACD97] L. Ambrosio, A. Coscia, and G. Dal Maso. Fine properties of functions with bounded deformation. Arch. Rational Mech. Anal., 139:201-238, 1997.

[AFP00] L. Ambrosio, N. Fusco, and D. Pallara. Functions of bounded variation and free discontinuity problems. Oxford Mathematical Monographs. Oxford University Press, New York, 2000.

[AG80] G. Anzellotti and M. Giaquinta. Existence of the displacement field for an elastoplastic body subject to Hencky's law and von Mises yield condition. Manuscripta Math., 32:101-136, 1980.

[Bab13] J.-F. Babadjian. Traces of functions of bounded deformation. Indiana Univ. Math. J., to appear, preprint 2013.

[BCDM98] G. Bellettini, A. Coscia, and G. Dal Maso. Compactness and lower semicontinuity properties in $\operatorname{SBD}(\Omega)$. Math. Z., 228:337351, 1998.

[BCM] D. Bourne, S. Conti, and S. Müller. Energy bounds for a compressed elastic film on a substrate. In preparation.

[BFM08] B. Bourdin, G. A. Francfort, and J.-J. Marigo. The variational approach to fracture. J. Elasticity, 91:5-148, 2008. 
[CCF $]$ A. Chambolle, S. Conti, and G. Francfort. Approximation of a brittle fracture energy with non-interpenetrating constraint. In preparation.

[CDM14] S. Conti, G. Dolzmann, and S. Müller. Korn's second inequality and geometric rigidity with mixed growth conditions. Calc. Var. Partial Differential Equations, 50:437-454, 2014.

[CFM05] S. Conti, D. Faraco, and F. Maggi. A new approach to counterexamples to $L^{1}$ estimates: Korn's inequality, geometric rigidity, and regularity for gradients of separately convex functions. Arch. Rat. Mech. Anal., 175:287-300, 2005.

[Cha03] A. Chambolle. A density result in two-dimensional linearized elasticity, and applications. Arch. Ration. Mech. Anal., 167:211233, 2003.

[Cha04] A. Chambolle. An approximation result for special functions with bounded deformation. J. Math. Pures Appl. (9), 83:929954, 2004.

[Cha05] A. Chambolle. Addendum to: "An approximation result for special functions with bounded deformation" [Cha04]. J. Math. Pures Appl. (9), 84:137-145, 2005.

[CS06] S. Conti and B. Schweizer. Rigidity and Gamma convergence for solid-solid phase transitions with $S O(2)$ invariance. Comm. Pure Appl. Math., 59:830-868, 2006.

[DM95] G. Dolzmann and S. Müller. Microstructures with finite surface energy: the two-well problem. Arch. Rat. Mech. Anal., 132:101$141,1995$.

[Eva98] L. C. Evans. Partial differential equations, volume 19 of Graduate Studies in Mathematics. American Mathematical Society, Providence, RI, 1998.

[FI14] M. Focardi and F. Iurlano. Asymptotic analysis of AmbrosioTortorelli energies in linearized elasticity. SIAM J. Math. Anal., 46:2936-2955, 2014.

[FJM02] G. Friesecke, R. D. James, and S. Müller. A theorem on geometric rigidity and the derivation of nonlinear plate theory from threedimensional elasticity. Comm. Pure Appl. Math., 55:1461-1506, 2002 . 
[FM98] G. A. Francfort and J.-J. Marigo. Revisiting brittle fracture as an energy minimization problem. J. Mech. Phys. Solids, 46:13191342, 1998.

[Iur14] F. Iurlano. A density result for GSBD and its application to the approximation of brittle fracture energies. Calc. Var. Partial Differential Equations, 51:315-342, 2014.

[KK11] B. Kirchheim and J. Kristensen. Automatic convexity of rank-1 convex functions. C. R. Math. Acad. Sci. Paris, 349:407-409, 2011.

[Koh82] R. V. Kohn. New integral estimates for deformations in terms of their nonlinear strains. Arch. Rational Mech. Anal., 78:131-172, 1982 .

[KT83] R. Kohn and R. Temam. Dual spaces of stresses and strains, with applications to Hencky plasticity. Appl. Math. Optim., 10:1-35, 1983.

[MSZ14] S. Müller, L. Scardia, and C. I. Zeppieri. Geometric rigidity for incompatible fields and an application to strain-gradient plasticity. Indiana Univ. Math. J., 63:1365-1396, 2014.

[Nit81] J. A. Nitsche. On Korn's second inequality. RAIRO Anal. Numér., 15:237-248, 1981.

[NPW12] P. Neff, D. Pauly, and K.-J. Witsch. Maxwell meets Korn: a new coercive inequality for tensor fields in $\mathbb{R}^{N \times N}$ with squareintegrable exterior derivative. Math. Methods Appl. Sci., 35:65$71,2012$.

[Orn62] D. A. Ornstein. A non-inequality for differential operators in the $L^{1}$-norm. Arch. Rat. Mech. Anal., 11:40-49, 1962.

[Reš70] J. G. Rešetnjak. Estimates for certain differential operators with finite-dimensional kernel. Sibirsk. Mat. Z̆., 11:414-428, 1970.

[SFO08] B. Schmidt, F. Fraternali, and M. Ortiz. Eigenfracture: an eigendeformation approach to variational fracture. Multiscale Model. Simul., 7:1237-1266, 2008.

[Suq78] P.-M. Suquet. Sur un nouveau cadre fonctionnel pour les équations de la plasticité. C. R. Acad. Sci. Paris Sér. A-B, 286:A1129-A1132, 1978. 
[Suq79] P.-M. Suquet. Un espace fonctionnel pour les équations de la plasticité. Ann. Fac. Sci. Toulouse Math. (5), 1:77-87, 1979.

[Tem83] R. Temam. Problèmes mathématiques en plasticité, volume 12 of Méthodes Mathématiques de l'Informatique. Gauthier-Villars, Montrouge, 1983.

[Tin72] T. W. Ting. Generalized Korn's inequalities. Tensor (N.S.), 25:295-302, 1972. Commemoration volumes for Prof. Dr. Akitsugu Kawaguchi's seventieth birthday, Vol. II.

[TS81] R. Temam and G. Strang. Functions of bounded deformation. Arch. Rational Mech. Anal., 75:7-21, 1980/81. 